Contributions to Game Theory and Management, XIII, 132-141

\title{
Models of Optimal Control in Tullock Rent-Seeking Game *
}

\author{
Denis N. Fedyanin \\ V.A.Trapeznikov Institute of Control Sciences, \\ Laboratory $5 \%$, \\ Profsoyuznaya str. 65, Moscow, Russia \\ E-mail: dfedyanin@inbox.ru \\ $W W W$ home page: www.ipu.ru/en
}

\begin{abstract}
The paper constructs and investigates the models of the optimal control in the Tullock rent-seeking game. There are two types of control in the paper: an unlimited, but expensive resource, and a cheap, but an infinitely small resource. Before the game starts, players discuss parameters of the game, and then choose their strategies simultaneously and independently, competing for better rent. We consider two types of players and two types of communication and analyze combinations.
\end{abstract}

Keywords: optimal control, Tullock rent-seeking game, parametrized equilibrium, beliefs

\section{Introduction}

Players discuss competition parameters for a prize and compete by making costly investments and choosing their strategies simultaneously and independently. Tullock introduced his model to describe how such players make decisions, but only if there were no negotiations and that the game parameters were common knowledge (Tullock, 1980).

Previous results and this paper (Fedyanin, 2020) incorporates utility functions from the Tullock rent-seeking game but pay attention to uncertainty and optimal control of players' beliefs. It provides tools to enrich investigations when players might have different initial beliefs, types, and protocols of preliminary negotiations before the game start (Aumann, 1999). Previous investigations (Fedyanin, 2019) introduced a model for the communication and results of belief interactions among players. We considered two types of players and two types of communication, and we analyzed all possible combinations. It leads us to the four unique combinations of types and communications for analysis. We have suggested epistemic models for all of them and calculated equilibriums for the first three of them.

- Game 1 is a classic Tullock rent-seeking game with common knowledge about the parameters. The optimal control is to control the true values of parameters. We consider this case as the simplest for comparison with other controls.

- Every player in Game 2 believes that all other players' beliefs and her beliefs about the values of the parameters coincide, and it is common knowledge.

- Game 3 assumes a consensus among players. Though players have initial beliefs, they change their beliefs to come to a single belief in a consensus. Though some expressions are very similar to those in Game 1, the control differs since we have to consider the influences.

\footnotetext{
* The paper was partially supported by the RSF grant 16-19-10609.
} 
- Every player in Game 4 knows all others' beliefs but believes that only his belief is the true one. We do not investigate the optimal problem for this game since we could not find an equilibrium.

In the paper, we present the corresponding formal models for each game with the formal description of the equilibriums conditions. These conditions are the systems of equations.

This system is infinite and might require complicated analysis, but it has compactness for the Games 1-4. It means that there is only a finite number of equivalent strategies.

The sizes of the corresponding systems are the following.

- The system is the same as the equation for the Nash equilibrium. Thus, $n$ equations for Game 1.

- The system includes one system of $n$ variables for each player, thus $n$ systems of $n$ equations for the Game 2.

- Though initial beliefs of players might be different - the consensus reduces the system to a modification of the Game 1. If there is no consensus and the players do not change their beliefs than the system becomes the same as for Game 4 . Thus Game 1 and Game 3 might be examples of Game 3 if we do not assume consensus and require beliefs coincide with the real values of parameters. So, it is $n$ equations for Game 3 .

- It is $\mathrm{n}$ equations for the Game 4.

Given these results, the paper focus on optimal control of beliefs about parameters. The optimal control problem in this paper is an optimization problem, where

- criteria of optimization is a function of strategies of players at the equilibria

- control is a parameter of the game, which is the parameter of the players' strategies at the equilibria.

We analyze the best way of spending a tiny amount of a resource to change the given beliefs and parameters of the game. Research plans to find an equilibrium for a given informational structure and calculate the partial derivatives. The most substantial partial derivative shows a belief or a parameter of the game, which is the first to apply control. We start from the straightforward models to show how the control algorithm works and proceed with the more sophisticated. We also provide examples.

The paper uses the following known results:

1. the formulation of the Tullock competition and expression for equilibria (Tullock, 1980),

2. the concept of beliefs (Harsanyi, 1967),

3. the concept of reflection game (Novikov et al. 2014),

4. the idea and formal model of weakening common knowledge required for the game by classifying the interaction of players into four combinations (Fedyanin, 2019),

5. the method to apply such weakening to a game and application to the Tullock competition (Fedyanin, 2020),

6. the idea and method of the linearization to find a maximum of the criteria and solve of control problem (Neudecker et al., 1988), 
7. the methods to find global optimum with restrictions (Beavis et al. 1990; Curtis, 2015).

Previous results cover a full chain of steps from 1 to 5 , and the literature describes 6 and 7. This paper applies methods from 6 and 7 to step 5 . These results are new.

This paper focuses on control problems that are a reasonable step forward from previous investigations. We introduce two classes of optimization:

- an infinitely large amount of control resource, but there are quadratic expenses for using it. We suggest the Laplace method to find a global maximum.

- an infinitely small amount of control resource; thus, we can linearize and apply all control to the control parameter, which derivative is greater than others.

\section{Game}

We consider Tullock rent-seeking game with uncertainty. There are applications: competition for monopoly rents, investments in $\mathrm{R} \& \mathrm{D}$, competition for a promotion/bonus, political contests. A formal model is as the following.

Reflective version of Tullock rent-seeking game $\Gamma_{I}$ is a game described by the following tuple:

$$
\Gamma_{I}=\left\{N,\left(X_{i}\right)_{i \in N}, f_{i}(\cdot)_{i \in N}, I\right\}
$$

where

- $N=\{1, \ldots, n>2\}$ is a set of players,

- $X=\left\{X_{1}, \ldots, X_{n}\right\}$ is a set of strategies of players, where $X_{i}=\left\{x_{i} \geq 0\right\}$ is a set of avalable strategies for the player $i$,

- $F=\left\{f_{1}, \ldots, f_{n}\right\}$ - is a set of the utility functions such that

$$
f_{i}\left(x_{1}, \ldots, x_{n}, \alpha, M, n\right)=\frac{x_{i}^{\alpha}}{\sum_{j \in N} x_{j}^{\alpha}} M-x_{i},
$$

where the restrictions on the parameters are $0<\alpha<1 \leq M$.

An informational structure is a way to model uncertainty by a tree where a belief of an player is a node in the tree. This tree is infinite in a general case.

Information structure is represented by a tree. We denote

- $(M, \alpha, n)_{a_{1}, \ldots, a_{k}}$ beliefs of an player $a_{1}$ about the belief of player $a_{2} \ldots$ about player $a_{k}$ about the values of $(M, \alpha, n) . I=\left\{(M, \alpha, n)_{a_{1}, \ldots, a_{k}} \forall a_{1}, \ldots, a_{k} \in N\right\}$. We denote $\left(M_{a}, \alpha_{a}, n_{a}\right)=(M, \alpha, n)_{a}$. See Fig. 1 .

- $x_{a_{1}, \ldots, a_{k}}$ a strategy chosen by an image of player $a_{k}$ in a beliefs of an player $a_{k-1} \ldots$ in beliefs of an player $a_{1}$. We assume that $x_{a_{1}, \ldots, a_{k}, j} \in X_{j}$

The equilibrium is a set of strategies of all images of players iff

$$
\begin{gathered}
x_{a_{1}, \ldots, a_{k}, i}= \\
B R_{i}\left((M, \alpha, n)_{a_{1}, \ldots, a_{k}}, x_{a_{1}, \ldots, a_{k}, 1}, \ldots, x_{a_{1}, \ldots, a_{k}, i-1}, \ldots, x_{a_{1}, \ldots, a_{k}, i+1}, \ldots, x_{a_{1}, \ldots, a_{k}, n}\right),
\end{gathered}
$$

where $B R_{i}$ is the best response of the player $i$ to the fixed strategies of other players with values of parameter according to player's beliefs. 


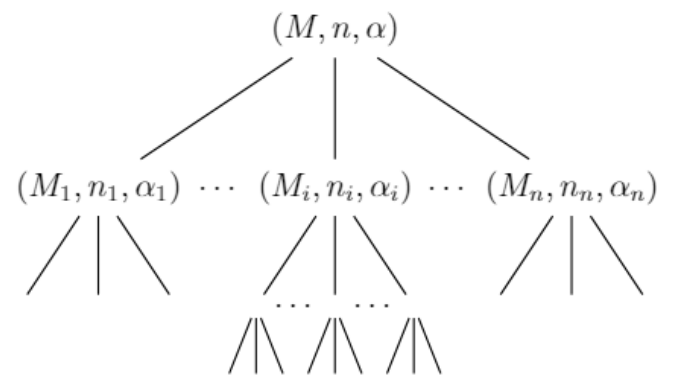

Fig. 1. An example of an informational structure. It is similar to Harsanyie types approach (Harsanyi, 1967/68)

We introduced four Games and have investigated the first three of them.

In Game 1, there is a common knowledge, and we have to find a solution for the system of the best responses (BR) of the players.

$$
x_{1}^{*}=B R_{1}\left(x_{-1}^{*}, M, n, \alpha\right) ; \ldots ; x_{n}^{*}=B R_{n}\left(x_{-n}^{*}, M, n, \alpha\right) .
$$

This solution gives us equilibrium.

In Game 2, players cannot communicate. A brief example of this model is the following. Let there are Ann and Bob. Ann watches the TV channel, and there is a claim that there is a storm nearby. She could think that it is such important news that everyone should know it. Bob does not know anything about the storm and thinks that nobody thinks that there is a storm know. Both of them are wrong in detail but make actions as they are right. We have to find a solution for the system of the best responses (BR) of the players.

$$
\begin{gathered}
x_{1}^{*}=B R\left(x_{-1}^{*}, M, n, \alpha\right) ; \ldots ; x_{n}^{*}=B R\left(x_{-n}^{*}, M, n, \alpha\right) ; \\
x_{1}^{* 1}=B R\left(x_{-1}^{* 1}, M_{1}, n_{1}, \alpha_{1}\right) ; \ldots ; x_{n}^{* 1}=B R\left(x_{-n}^{* 1}, M_{1}, n_{1}, \alpha_{1}\right) ; \\
x_{1}^{* j}=B R\left(x_{-1}^{* j}, M_{j}, n_{j}, \alpha_{j}\right) ; \ldots ; x_{n}^{* j}=B R\left(x_{-n}^{* j}, M_{j}, n_{j}, \alpha_{j}\right) ; \\
x_{1}^{* n}=B R\left(x_{-1}^{* n}, M_{n}, n_{n}, \alpha_{n}\right) ; \ldots ; x_{n}^{* n}=B R\left(x_{-n}^{* n}, M_{n}, n_{n}, \alpha_{n}\right) ; \\
x_{1}^{*}=x_{1}^{* 1} ; \ldots ; x_{n}^{*}=x_{n}^{* n} .
\end{gathered}
$$

This solution gives us equilibrium.

In Game 3, players are allowed to communicate and reach consensus. There could be communication between players, and they can communicate according to the de Groot model (DeGroot, 1974; Gubanov et al., 2009). There is no difference if the existence of such communication to the common knowledge among all players, or it is not.

$$
M^{*}=\sum_{i \in N} w_{i}^{M} M_{i} ; \alpha^{*}=\sum_{i \in N} w_{i}^{\alpha} \alpha_{i} ; n^{*}=\sum_{i \in N} w_{i}^{n} n_{i},
$$

where $w_{i}^{M}, w_{i}^{\alpha}, w_{i}^{n}$ are the final influences (Gubanov et al., 2009) of the player $i$ on a social network consensus opinion about $M, \alpha, n$ 
We have to find a solution for the system of the best responses (BR) of the players.

$$
\begin{aligned}
& x_{1}^{*}=B R\left(x_{-1}^{*}, \sum_{i} w_{i}^{M} M_{i}, \sum_{i} w_{i}^{n} n, \sum_{i} w_{i}^{\alpha} \alpha\right) ; \\
& x_{n}^{*}=B R\left(x_{-n}^{*}, \sum_{i} w_{i}^{M} M_{i}, \sum_{i} w_{i}^{n} n, \sum_{i} w_{i}^{\alpha} \alpha\right) .
\end{aligned}
$$

This solution gives us equilibrium.

\section{Optimal Control}

We consider two types of optimal control: local and global, and the restrictions on control.

\subsection{Unlimited, but Expensive Control Resource}

We look for optimal control in the form of the maximum number of strategies of players reduced by the control's quadratic expenses. The criteria for the optimization are the following.

Game 1 The criteria for Game 1 is

$$
\begin{gathered}
F=\sum_{j} x_{j}-\left(\left(M-M_{0}\right)^{2}+\left(\alpha-\alpha_{0}\right)^{2}+\left(n-n_{0}\right)^{2}\right), \\
F=\frac{n-1}{n} \alpha M-\left(\left(M-M_{0}\right)^{2}+\left(\alpha-\alpha_{0}\right)^{2}+\left(n-n_{0}\right)^{2}\right) . \\
F=\frac{n-1}{n} \alpha M-\left(\left(M-M_{0}\right)^{2}+\left(\alpha-\alpha_{0}\right)^{2}+\left(n-n_{0}\right)^{2}\right) . \\
\frac{\partial}{\partial M} F=\frac{n-1}{n} \alpha-2\left(M-M_{0}\right)=0 \\
\frac{\partial}{\partial \alpha} F=\frac{n-1}{n} M-2\left(\alpha-\alpha_{0}\right)=0 \\
\frac{\partial}{\partial n} F=\frac{2-n}{n^{2}} \alpha M-2\left(n-n_{0}\right)=0
\end{gathered}
$$

Hessian is

$$
\begin{gathered}
H(F)=\left(\begin{array}{c}
\frac{\partial^{2}}{\partial M^{2}} F=-2 ; \quad \frac{\partial^{2}}{\partial M \partial \alpha} F=-\frac{n-1}{n} ; \quad \frac{\partial^{2}}{\partial M \partial n} F=\frac{2-n}{n^{2}} \alpha \\
\frac{\partial^{2}}{\partial \alpha \partial M} F=-\frac{n-1}{n} ; \quad \frac{\partial^{2}}{\partial \alpha^{2}} F=-2 ; \quad \frac{\partial^{2}}{\partial \alpha \partial n} F=\frac{2-n}{n^{2}} M \\
\frac{\partial^{2}}{\partial n \partial M} F=\frac{2-n}{n^{2}} \alpha ; \quad \frac{\partial^{2}}{\partial n \partial \alpha} F=\frac{2-n}{n^{2}} M ; \quad \frac{\partial^{2}}{\partial n^{2}} F=-2 ;
\end{array}\right) ; \\
\operatorname{det} H(F)=\frac{2\left(M^{2} n(n-2)^{2}-\alpha M(n-1)(n-2)^{2}\right)+}{n^{5}} \\
\frac{2 n\left(4 \alpha^{2}+\left(\alpha^{2}+1\right) n^{2}-3 n^{4}-2 n^{3}-4 \alpha^{2} n\right)}{n^{5}} .
\end{gathered}
$$

For large enough $n$ Hessian is negative definite and thus for large enough $n$ there is a maximum of $\mathrm{F}$ if the gradient is zero. 
The zero points of the gradient are complicated, but there is a simple condition on $M$ and $\alpha$ at the maximum.

$$
\frac{\alpha}{M}=\frac{M-M_{0}}{\alpha-\alpha_{0}} .
$$

Game 2 The criteria for Game 2 is

$$
\begin{gathered}
F=\sum_{j} x_{j}-\sum_{j}\left(\left(M_{j}-M_{j 0}\right)^{2}+\left(\alpha_{j}-\alpha_{j 0}\right)^{2}+\left(n_{j}-n_{j 0}\right)^{2}\right), \\
F=\sum_{k=1}^{m} \frac{M_{k}\left(n_{k}-1\right) \alpha_{k}}{n_{k}^{2}}-\sum_{j}\left(\left(M_{j}-M_{j 0}\right)^{2}+\left(\alpha_{j}-\alpha_{j 0}\right)^{2}+\left(n_{j}-n_{j 0}\right)^{2}\right) .
\end{gathered}
$$

If the Hessian is negative definite then the zeros of the gradient are local maximums as in the Game 1. In this case there are similar conditions on some parameters in a maximum for any $k \in N$ :

$$
\frac{\alpha_{k}}{M_{k}}=\frac{M_{k}-M_{k 0}}{\alpha_{k}-\alpha_{k 0}}
$$

We suggest that for large enough $n_{k}$ Hessian is negative definite since the determinant of a block diagonal matrix is a product of the determinants of its blocks. It matters since the Hessian here is a block diagonal one.

Game 3 The criteria for Game 3 is

$$
\begin{gathered}
F=\sum_{j} x_{j}-\sum_{j}\left(\left(M_{j}-M_{j 0}\right)^{2}+\left(\alpha_{j}-\alpha_{j 0}\right)^{2}+\left(n_{j}-n_{j 0}\right)^{2}\right)- \\
\sum_{j}\left(\left(w_{j}^{M}-w_{j 0}^{M}\right)^{2}+\left(w_{j}^{\alpha}-w_{j 0}^{\alpha}\right)^{2}+\left(w_{j}^{n}-w_{j 0}^{n}\right)^{2}\right) . \\
F=\frac{\sum_{j=1}^{m} n_{j} w_{j}^{n}-1}{\sum_{j=1}^{m} n_{j} w_{j}^{n}} \sum_{j=1}^{m} w_{j}^{\alpha} \alpha_{j} \sum_{j=1}^{m} M_{j} w_{j}^{M}- \\
\frac{1}{r} \sum_{j}\left(\left(M_{j}-M_{j 0}\right)^{2}+\left(\alpha_{j}-\alpha_{j 0}\right)^{2}+\left(n_{j}-n_{j 0}\right)^{2}\right)- \\
\frac{1}{r} \sum_{j}\left(\left(w_{j}^{M}-w_{j 0}^{M}\right)^{2}+\left(w_{j}^{\alpha}-w_{j 0}^{\alpha}\right)^{2}+\left(w_{j}^{n}-w_{j 0}^{n}\right)^{2}\right),
\end{gathered}
$$

where

$$
M^{*}=\sum_{j=1}^{m} M_{j} w_{j}^{M} \alpha^{*}=\sum_{j=1}^{m} \alpha_{j} w_{j}^{\alpha} ; n^{*}=\sum_{j=1}^{m} n_{j} w_{j}^{n} .
$$

The gradient will be zero if

$$
\begin{aligned}
\frac{n^{*}-1}{n^{*}} \alpha^{*} w_{k}^{M} & =2\left(M_{k}-M_{k 0}\right) ; \frac{n^{*}-1}{n^{*}} M^{*} w_{k}^{\alpha}=2\left(\alpha_{k}-\alpha_{k 0}\right) ; \\
& \frac{2-n^{*}}{\left(n^{*}\right)^{2}} \alpha^{*} M^{*} w_{k}^{n}=2\left(n_{k}-n_{k 0}\right) ;
\end{aligned}
$$




$$
\begin{aligned}
\frac{n^{*}-1}{n^{*}} \alpha^{*} M_{k}= & 2\left(w_{k}^{M}-w_{k 0}^{M}\right) ; \frac{n^{*}-1}{n^{*}} \alpha_{k} M^{*}=2\left(w_{k}^{\alpha}-w_{k 0}^{\alpha}\right) ; \\
& \frac{2-n^{*}}{\left(n^{*}\right)^{2}} \alpha^{*} M^{*}=2\left(w_{k}^{n}-w_{k 0}^{n}\right) .
\end{aligned}
$$

There are restrictions: $0<\alpha_{k}<1,1<M, 0<w_{i}^{M}<1,0<w_{i}^{\alpha}<1,0<w_{i}^{n}<$ $1, \sum_{j} w_{j}^{M}=1, \sum_{j} w_{j}^{\alpha}=1, \sum_{j} w_{j}^{n}=1$. Restrictions assumes that we should use Lagrange multiplier in general case or Boarded Hessian. These methods are well known, though complicated for our case.

The Hessian can also be applied in the next section if we use not linear but quadratic approximation since the corresponding Taylor series include Hessian.

\subsection{Cheap, but Infinitely Small Control Resource}

The criteria $F$ consists of continuous and discrete variables because usually, the number of players is discrete. However, if the number is large enough, we can approximate it by a constant value.

If there is only a small amount of a resource, we cannot apply the approach that we use for unlimited resources. In this case, we rewrite the criteria as $F=\sum_{j} x_{j}$ with the following restrictions: $M^{2}+\alpha^{2}+n^{2} \leq R$ for Game 1,

$$
\sum_{j}\left(\left(M_{j}-M_{j 0}\right)^{2}+\left(\alpha_{j}-\alpha_{j 0}\right)^{2}+\left(n_{j}-n_{j 0}\right)^{2}\right) \leq R
$$

for Game 2,

$$
\begin{gathered}
\sum_{j}\left(\left(M_{j}-M_{j 0}\right)^{2}+\left(\alpha_{j}-\alpha_{j 0}\right)^{2}+\left(n_{j}-n_{j 0}\right)^{2}\right)- \\
\sum_{j}\left(\left(w_{j}^{M}-w_{j 0}^{M}\right)^{2}+\left(w_{j}^{\alpha}-w_{j 0}^{\alpha}\right)^{2}+\left(w_{j}^{n}-w_{j 0}^{n}\right)^{2}\right) \leq R
\end{gathered}
$$

for Game 3.

When $R$ is small enough we can calculate an opimal solution having derivatives by choing the maximum of derivatives like

$$
\max \left(\frac{\partial}{\partial M} \sum_{j} x_{j}, \frac{\partial}{\partial \alpha} \sum_{j} x_{j}, \frac{\partial}{\partial n} \sum_{j} x_{j}\right)
$$

and apply all control to an argmax.

Game 1 Linearization leads to the following expression for the linear approximation of the maximum criteria, which can be reached by an amount of the resource $R$.

$$
R \max \left(\frac{n_{0}-1}{n_{0}} \alpha_{0}, \frac{n_{0}-1}{n_{0}} M_{0}, \frac{n_{0}-2}{n_{0}^{2}} \alpha_{0} M_{0}\right) .
$$

Given $0<\alpha<1 \leq M$ the expressions leads to a simple optimal control rule that is always spend all resource to increase $\alpha$ up to $\alpha=\alpha_{0}+R$ if $\alpha_{0}<1-R$. The increase of the criteria $\mathrm{F}$ will be approximately

$$
\frac{n_{0}-1}{n_{0}} R M_{0}
$$


since the largest derivative is

$$
\frac{\partial}{\partial \alpha} \sum_{j} x_{j}=\frac{n-1}{n} M
$$

because

$$
\begin{gathered}
R \max \left(\frac{n_{0}-1}{n_{0}} \alpha_{0}, \frac{n_{0}-1}{n_{0}} M_{0}, \frac{n_{0}-2}{n_{0}^{2}} \alpha_{0} M_{0}\right)= \\
R \frac{n_{0}-1}{n_{0}} \max \left(\alpha_{0}, M_{0}, \frac{n_{0}-2}{n_{0}-1} \alpha_{0} M_{0}\right)=R \frac{n_{0}-1}{n_{0}} \max \left(\alpha_{0}, M_{0}\right)= \\
\frac{n_{0}-1}{n_{0}} R M_{0},
\end{gathered}
$$

if $\alpha_{0}>1-R$. The optimal control is to spend resource $1-\alpha_{0}$ to increase $\alpha$ up to 1 , and spend the rest to the increasing $M$ up to $M=M_{0}+R-1+\alpha_{0}$. The increase of the criteria $\mathrm{F}$ will be approximately

$$
\frac{n_{0}-1}{n_{0}}\left(\left(1-\alpha_{0}\right) M_{0}+\left(R-1+\alpha_{0}\right) \alpha_{0}\right) .
$$

Game 2 We can use the following expression for the linear approximation of the maximum of criteria, which can be reached by an amount of the resource $R$.

$$
\begin{gathered}
R \max _{k \in N}\left(\frac{\left(n_{k 0-1}\right) \alpha_{k 0}}{n_{k 0}^{2}}, \frac{M_{k 0}\left(n_{k 0}-1\right)}{n_{k 0}^{2}}, \frac{M_{k 0}\left(n_{k 0}-2\right) \alpha_{k 0}}{n_{k 0}{ }^{3}}\right)= \\
R \max _{k \in N}\left(\frac{\left(n_{k 0}-1\right) \alpha_{k 0}}{n_{k 0}^{2}}, \frac{M_{k 0}\left(n_{k 0}-1\right)}{n_{k 0}^{2}}\right)=R \max _{k \in N} \frac{M_{k 0}\left(n_{k 0}-1\right)}{n_{k 0}^{2}} \approx R \max _{k \in N} \frac{M_{k 0}}{n_{k 0}} .
\end{gathered}
$$

if $n-\sum_{j} \alpha_{j 0}>R$ then the algorithm of the optimal control is the following.

1. Assign $M:=N$.

2. Choose an player from $M$ with maximum $M_{k 0}\left(n_{k 0}-1\right) / n_{k 0}^{2}$ among all players in $M$. Denote such player by $j$

3. If $1-\alpha_{j 0}$ is larger then $R$ then spend $R$ to increase $\alpha_{j}$ up to $\alpha_{j}=\alpha_{j 0}+R$ and exit.

4. If $1-\alpha_{j 0}$ is smaller than $R$ or equals it then spend $1-\alpha_{j 0}$ to increase $\alpha_{j}$ up to $\alpha_{j}=1$,

5. Assign $R:=R-1+\alpha_{j 0}$

6. Exclude $j$ from $M$, and if $M$ is not empty and $R>0$ go to step b.

Game 3 We can use the following expression for the linear approximation of the maximum of criteria, which can be reached by an amount of the resource $R$.

$$
R \frac{n^{*}-1}{n^{*}} \max _{k \in N}\left(\alpha^{*} w_{k}^{M}, M w_{k}^{\alpha}, \frac{n^{*}-2}{n^{*}-1} \alpha^{*} M^{*} w_{k}^{n}, \alpha^{*} M_{k}, \alpha_{k} M^{*}, \frac{n^{*}-2}{n^{*}-1} \alpha^{*} M^{*}\right),
$$

where

$$
M^{*}=\sum_{j=1}^{m} M_{j} w_{j}^{M} \alpha^{*}=\sum_{j=1}^{m} \alpha_{j} w_{j}^{\alpha} ; n^{*}=\sum_{j=1}^{m} n_{j} w_{j}^{n} .
$$




\section{Conclusion}

The paper provides the solutions for special cases of the optimal problems for Tullock rent-seeking game with preliminary negotiations when there are unlimited large or infinitely small amounts of control resources. The solution for the stubborn players with communication (Game 4) is unknown since there is no known expression for equilibrium. There are known straight, but complicated ways to solve the optimization problem in general, but the investigation obtained simple expressions for some critical cases. This paper makes an essential step at the transition from the previously obtained expressions for parametrized equilibrium to the solved control problems.

\section{Acknowlegments.}

The paper was partially supported by the RSF grant 16-19-10609.

\section{Appendix. Parametrized equilibriums}

\section{Game 1. Players with common knowledge}

Actions of players are

$$
x_{i}^{*}=\frac{n-1}{n^{2}} \alpha M ; \forall i \in N .
$$

Furthermore, the following derivatives will be monotonicity could be found by an analysis of For short, we will use $x_{i}=x_{i}^{*}$.

$$
\begin{gathered}
\sum_{j} x_{j}^{*}=\frac{n-1}{n} \alpha M \\
\frac{\partial}{\partial M} \sum_{j} x_{j}=\frac{n-1}{n} \alpha ; \frac{\partial}{\partial \alpha} \sum_{j} x_{j}=\frac{n-1}{n} M ; \frac{\partial}{\partial n} \sum_{j} x_{j}=\frac{2-n}{n^{2}} \alpha M
\end{gathered}
$$

\section{Game 2. Players without communication}

Actions of players are

$$
x_{i}^{*}=\frac{n_{i}-1}{n_{i}^{2}} \alpha_{i} M_{i} .
$$

Moreover, monotonicity abe found by an analysis of the following derivatives.

$$
\begin{gathered}
\sum_{i} x_{i}^{*}=\sum_{k=1}^{m} \frac{M_{k}\left(n_{k}-1\right) \alpha_{k}}{n_{k}^{2}} \\
\frac{\partial}{\partial M_{k}} \sum_{i} x_{i}^{*}=\frac{\left(n_{k}-1\right) \alpha_{k}}{n_{k}^{2}} ; \frac{\partial}{\partial \alpha_{k}} \sum_{i} x_{i}^{*}=\frac{M_{k}\left(n_{k}-1\right)}{n_{k}{ }^{2}} ; \\
\frac{\partial}{\partial n_{k}} \sum_{i} x_{i}^{*}=\frac{M_{k}\left(2-n_{k}\right) \alpha_{k}}{n_{k}{ }^{3}}
\end{gathered}
$$




\section{Game 3. Players with communication and consensus}

Actions of players are

$$
x_{i}^{*}=\frac{n^{*}-1}{\left(n^{*}\right)^{2}} \alpha^{*} M^{*} .
$$

Moreover, the following derivatives are useful for the analysis of the monotonicity.

$$
\begin{gathered}
\frac{\partial}{\partial M_{k}} \sum_{j} x_{j}=\frac{n^{*}-1}{n^{*}} \alpha^{*} w_{k}^{M} ; \frac{\partial}{\partial \alpha_{k}} \sum_{j} x_{j}=\frac{n^{*}-1}{n^{*}} M^{*} w_{k}^{\alpha} ; \\
\frac{\partial}{\partial n_{k}} \sum_{j} x_{j}=\frac{2-n^{*}}{\left(n^{*}\right)^{2}} \alpha^{*} M^{*} w_{k}^{n} \\
\frac{\partial}{\partial w_{k}^{M}} \sum_{j} x_{j}=\frac{n^{*}-1}{n^{*}} \alpha^{*} M_{k} ; \frac{\partial}{\partial w_{k}^{\alpha}} \sum_{j} x_{j}=\frac{n^{*}-1}{n^{*}} \alpha_{k} M * ; \\
\frac{\partial}{\partial w_{k}^{n}} \sum_{j} x_{j}=\frac{2-n^{*}}{\left(n^{*}\right)^{2}} \alpha^{*} M^{*}
\end{gathered}
$$

\section{References}

Tullock, G. (1980). Efficient rent-seeking. In Buchanan, J.; Tollison, R.; Tullock, G. (eds.). Toward a theory of the rent-seeking society. College Station: Texas A \& M Press, 97-112.

Aumann, R. J. (1999). Interactive epistemology I: Knowledge. International Journal of Game Theory, 28(3), 263-300.

Harsanyi, J. C. (1967-1968). Games with Incomplete Information Played by Bayesian Players, I-III. Management Science, 14(3), 159-183 (Part I), 14(5), 320-334 (Part II), 14(7), 486-502 (Part III).

Novikov, D., Chkhartishvili, A. (2014). Reflexion Control: Mathematical models.// Series: Communications in Cybernetics, Systems Science, and Engineering (Book 5). CRC Press. P. 298.

DeGroot, M. H. (1974). Reaching a Consensus. Journal of American Statistical Association, 69(345), 118-121.

Fedyanin, D. (2019). Information Control in Reflexive Games with players Preliminary Informational Interactions. Proceedings of the 1st International Conference on Control Systems, Mathematical Modeling, Automation and Energy Efficiency (SUMMA2019, Lipetsk. IEEE, pp. 188-193.

Gubanov, D. A., Novikov, D. A., Chkhartishvili, A. G. (2009/2011). Informational influence and information control models in social networks. Probl. Upr., 2009, 5, 28-35; Autom. Remote control, 2011, 72(7), 1557-1567.

Fedyanin, D. (2020). Reflexive and epistemic properties of the Tullock rent-seeking game. Frontiers of Dynamic Games. Springer (in press).

Beavis, B., Dobbs, I. M. (1990). Static Optimization. Optimization and Stability Theory for Economic Analysis. New York: Cambridge University Press. P. 40.

Neudecker, H., Magnus, J.R. (1988). Matrix Differential Calculus with Applications in Statistics and Econometrics. New York: John Wiley\& Sons. P. 136.

Curtis, F. E., Que, X. (2015). A Quasi-Newton Algorithm for Nonconvex, Nonsmooth Optimization with Global Convergence Guarantees. Mathematical Programming Computation, 7(4), 399-428. 